\title{
BMJ Open Association of myocardial fibrosis detected by late gadolinium-enhanced MRI with clinical outcomes in patients with diabetes: a systematic review and meta-analysis
}

\author{
Zhi Yang, ${ }^{1,2}$ Rong Xu, ${ }^{1}$ Jia-rong Wang, ${ }^{3}$ Hua-yan Xu, ${ }^{1}$ Hang Fu, ${ }^{1}$ Ling-jun Xie, ${ }^{1}$ \\ Meng-xi Yang, ${ }^{3}$ Lu Zhang, ${ }^{1}$ Ling-yi Wen, ${ }^{1}$ Hui Liu, ${ }^{1}$ Hong Li, ${ }^{4}$ Zhi-gang Yang, ${ }^{3}$ \\ Ying-kun Guo (D) 1
}

To cite: Yang Z, Xu R,

Wang J, et al. Association of myocardial fibrosis detected by late gadolinium-enhanced MRI with clinical outcomes in patients with diabetes: a systematic review and meta-analysis. BMJ Open 2022;12:e055374. doi:10.1136/ bmjopen-2021-055374

- Prepublication history and additional supplemental material for this paper are available online. To view these files, please visit the journal online (http://dx.doi.org/10.1136/ bmjopen-2021-055374).

Z-gY and Y-kG contributed equally.

$\mathrm{ZY}$ and $\mathrm{RX}$ are joint first authors.

Received 11 July 2021

Accepted 17 December 2021

Check for updates

(c) Author(s) (or their employer(s)) 2022. Re-use permitted under CC BY-NC. No commercial re-use. See rights and permissions. Published by BMJ.

For numbered affiliations see end of article.

Correspondence to Dr Ying-kun Guo; gykpanda@163.com

\section{ABSTRACT}

Objective This meta-analysis assessed the associations of myocardial fibrosis detected by late gadoliniumenhanced (LGE)-MRI with the risk of major adverse cardiac and cerebrovascular events (MACCEs) and major adverse cardiac events (MACEs) in patients with diabetes.

Design Systematic review and meta-analysis reported in accordance with the guidelines of the Meta-analysis of Observational Studies in Epidemiology statement.

Data sources We searched the Medline, Embase and Cochrane by Ovid databases for studies published up to 27 August 2021.

Eligibility criteria Prospective or respective cohort studies were included if they reported the HR and 95\% Cls for MACCEs/MACEs in patients with either type 1 or 2 diabetes and LGE-MRI-detected myocardial fibrosis compared with patients without LGE-MRI-detected myocardial fibrosis and if the articles were published in the English language.

Data extraction and synthesis Two review authors independently extracted data and assessed the quality of the included studies. Pooled HRs and 95\% Cls were analysed using a random effects model. Heterogeneity was assessed using forest plots and $\mathrm{I}^{2}$ statistics.

Results Eight studies with 1121 patients with type 1 or type 2 diabetes were included in this meta-analysis, and the follow-up ranged from 17 to 70 months. The presence of myocardial fibrosis detected by LGE-MRI was associated with an increased risk for MACCEs (HR: 2.58; $95 \% \mathrm{Cl} 1.42$ to $4.71 ; p=0.002$ ) and MACEs (HR: $5.28 ; 95 \% \mathrm{Cl} 3.20$ to $8.70 ; p<0.001$ ) in patients with diabetes. Subgroup analysis revealed that ischaemic fibrosis detected by LGE was associated with MACCEs ( $\mathrm{HR} 3.80,95 \% \mathrm{Cl} 2.38$ to $6.07 ; p<0.001)$ in patients with diabetes.

Conclusions This study demonstrated that ischaemic myocardial fibrosis detected by LGE-MRI was associated with an increased risk of MACCEs/MACEs in patients with diabetes and may be an imaging biomarker for risk stratification. Whether LGE-MRI provides incremental prognostic information with respect to MACCEs/MACEs over risk stratification by conventional cardiovascular risk factors requires further study.

\section{Strengths and limitations of this study}

- This meta-analysis was performed in accordance with the guidelines of the Meta-analysis of Observational Studies in Epidemiology statement.

- All included studies were not community-based epidemiology research and came from developed countries.

- Reduced left ventricular ejection fraction and nonischaemic subgroup analyses were not performed due to the limited number of related studies.

\section{INTRODUCTION}

Diabetes is becoming a global healthcare problem, and it is estimated that there will be 693 million individuals with diabetes by 2045. ${ }^{1}$ Patients with diabetes have a higher prevalence of ischaemic myocardial fibrosis and non-ischaemic myocardial fibrosis than their non-diabetic counterparts, and the mechanism has been confirmed extensively. ${ }^{2-5}$ The phenotype of unrecognised ischaemic myocardial fibrosis in patients with diabetes was well studied and was associated with 4-8 folds increase in the risk of major adverse cardiac events (MACEs). ${ }^{2}{ }^{3}$ However, even without myocardial ischaemia, hyperglycaemia, oxidative stress and inflammation may lead to diffuse interstitial and non-ischaemic myocardial fibrosis in patients with diabetes. ${ }^{6-8}$ In addition, diffuse interstitial myocardial fibrosis can increase the risk of non-ischaemic myocardial fibrosis, and was associated with increased risk of left ventricular (LV) dysfunction in patients with diabetes. ${ }^{9} 10$ However, non-ischaemic myocardial fibrosis, may be a biomarker for risk stratification, has not been systematically characterised. $^{39}$ 
Among detectors of myocardial fibrosis, late gadolinium-enhanced MRI (LGE-MRI) is the most reliable tool for identifying and quantifying focal myocardial fibrosis in vivo and allows discrimination between ischaemic and non-ischaemic fibrosis without ionising radiation. ${ }^{11-13}$ LGE-MRI, a promising technique, can provide more histological information than unenhanced cardiac MRI to illuminate the complex pathophysiologic pathways of myocardial viability. ${ }^{3}$ While LGE-MRI is limited by its sensitivity and accuracy for detection of diffuse myocardial fibrosis, the role of T1-mapping MRI technique in quantifying myocardial fibrosis has been validated. ${ }^{12}{ }^{13}$ Furthermore, recent guidelines suggested that MRI may be considered an imaging technique for stratifying cardiovascular risk in patients with diabetes. ${ }^{14} 15$ This may highlight the role of LGE-MRI in the risk stratification of patients with diabetes.

Approximately $19 \%$ of asymptomatic patients with diabetes have myocardial fibrosis on LGE-MRI. ${ }^{2}$ Although several studies have demonstrated that focal myocardial fibrosis detected by LGE-MRI may predict MACEs in patients with diabetes, the prognostic value of focal myocardial fibrosis for major cardiac and cerebrovascular events (MACCEs) is unclear. ${ }^{3}{ }^{16-21}$ In addition, most previous studies were single-centre studies and have been limited by small numbers of events. Consequently, we performed a meta-analysis to assess the association of LV myocardial fibrosis detected by LGE-MRI with future MACCEs and MACEs in patients with diabetes.

\section{METHODS}

This meta-analysis was performed in accordance with the guidelines of the Meta-analysis of Observational Studies in Epidemiology statement. ${ }^{22} 23$

\section{Data sources and searches}

We searched the Ovid Medline, Ovid Embase and Ovid Cochrane Library databases to find eligible studies published up to 27 August 2021. The search strategy included the following keywords: "diabetes", "diabetes mellitus", "MR", "cardiac magnetic resonance", "CMR", "gadolinium", "LGE", "prognosis", "diagnosed", "predictor", and "death". The details of the search strategy used for Ovid are available in online supplemental tables S1-S3. In addition, only articles published in peer-reviewed journals and published in the English language were included.

\section{Study selection}

All articles were independently screened by two reviewers (ZY, RX), and any disagreement was resolved by consensus. The inclusion criteria were as follows: the design was a prospective or retrospective cohort study; the populations were patients with diabetes, and exposure to myocardial fibrosis was detected by LGE-MRI; the outcomes used composite endpoints including allcause mortality, cardiac and cerebrovascular disease, late coronary revascularisation, and hospitalisation for unstable angina; the study reported the HR and 95\% CIs and had $\geq 12$ months of follow-up. We excluded reviews, abstracts, animal studies, case reports and cross-sectional studies. Additionally, if the cases were reported more than once, we included the study with the most comprehensive information. The reviewers independently screened the titles first, then the abstracts, and finally the full texts.

\section{Data extraction and quality assessment}

We extracted the following data from each included study: author, year of publication, sample size, study design, age, LGE-MRI-detected myocardial fibrosis status, follow-up duration, outcome and HR (95\% CI). Additionally, we extracted the adjusted HR if the study reported the HR with adjustment models.

All of the included studies were prospective or retrospective cohort designs, and we used the Newcastle Ottawa Scale (NOS) to judge the quality of the studies, as this tool is usually used for evaluating the quality of cohort studies in meta-analyses. ${ }^{24}{ }^{25}$ The scale uses a maximum of nine points involving three factors: patient selection $(0-4$ points), comparability (0-2 points) and outcome (0-3 points). ${ }^{26}$ We categorised the quality of studies as low (0-3 scores), moderate (4-6 scores) and high (7-9 scores).

\section{Data synthesis and analysis}

In this meta-analysis, the outcome measure was the prevalence of future adverse cardiac and/or cerebrovascular events among diabetes patients with LGE-MRI-detected myocardial fibrosis compared with those without LGEMRI-detected myocardial fibrosis. We defined the primary endpoint as MACCEs, including myocardial infarction (MI), all-cause mortality, coronary and carotid revascularisation, heart failure, ventricular arrhythmias, unstable angina, cardiac and cerebrovascular death, and cerebrovascular disease. The secondary endpoints were MACEs, including all-cause mortality, cardiac death, MI, heart failure, unstable angina and ventricular arrhythmias. Additionally, the pattern of myocardial fibrosis was classified as ischaemic fibrosis or non-ischaemic fibrosis as described previously. ${ }^{3}$

We pooled the adjusted HRs with 95\% CIs using a random effects model. In addition, we calculated the annualised event rates by dividing the total events by the median follow-up periods. To analyse the heterogeneity of the included studies, we used forest plots and the $\mathrm{I}^{2}$ statistic. ${ }^{27}$ We assigned $\mathrm{I}^{2}$ values of $0 \sim 25 \%, \sim 50 \%, \sim 75 \%$ for low, medium and high heterogeneity of studies, respectively. Considering the heterogeneity of the included studies, we conducted sensitivity analyses by omitting one article to assess the influence of a single study. In particular, subgroup analyses were performed by outcome and the pattern of myocardial fibrosis. Additionally, a funnel plot was used to assess the publication bias of the included studies. ${ }^{28}$ The analyses were performed with Stata V.12 (StataCorp). P values were two sided, with a level of 0.05 considered significant. 


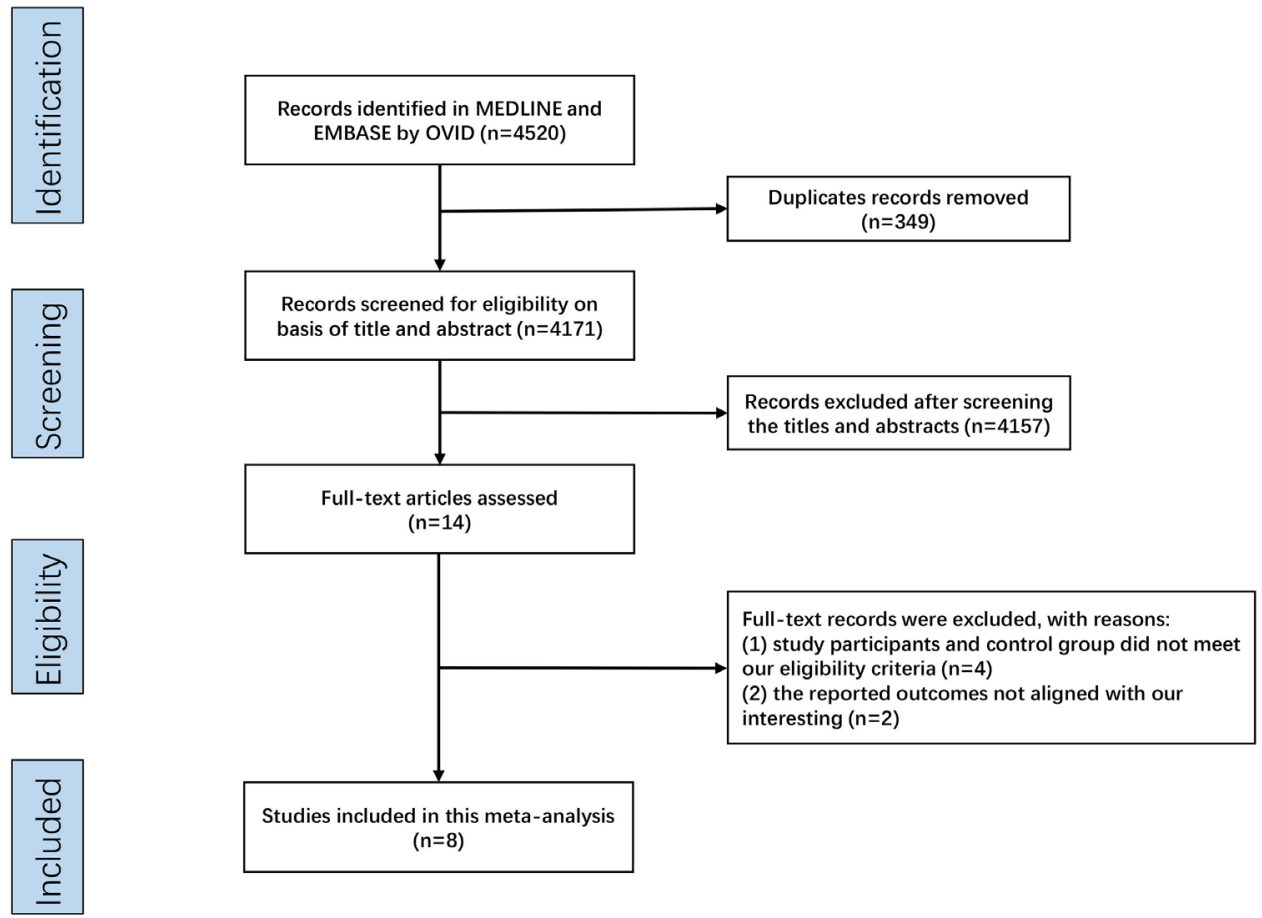

Figure 1 Flow chart of literature and study selection.

Patient and public involvement

No patient involved.

\section{RESULTS}

\section{Literature search}

Based on the selection strategy, we found 4520 citations. Of these, 349 duplicate studies were excluded. After screening the title and abstract, 14 articles remained for assessment of the full text. Six studies ${ }^{29-34}$ were excluded for the following reasons: studies without our outcome of interest, study populations did not meet our inclusion criteria, and studies did not report the HR. Ultimately, eight studies ${ }^{2}{ }^{316-21}$ fulfilled our inclusion criteria and were included in this meta-analysis (figure 1).

\section{Study characteristics}

In aggregate, 8 studies were analysed, including a total of 1121 patients with diabetes (median age ranging from 52 to $67 ; 67 \%$ were men) who underwent LGE-MRI and whose follow-up duration ranged from 17 to 70 months. Across the 8 studies, 6 articles ${ }^{217-21}$ reported the duration of diabetes, and the mean duration of diabetes was 15 years. A total of 6 studies ${ }^{231619-21}$ reported the LV ejection fraction, and the mean LV ejection fraction was $57.78 \%$. The presence of LGE-MRI-detected myocardial fibrosis was evaluated by visual analysis in six studies. ${ }^{2318-21}$ All of the included studies reported multiple clinical outcomes. The main characteristics of the included articles are shown in table 1 .

Among the eight selected studies, six studies ${ }^{16-21}(75 \%)$ were conducted in a single centre (Germany, $n=2$; USA, $\mathrm{n}=2$; Japan, $\mathrm{n}=2$ ), and two studies ${ }^{2}{ }^{3}$ were performed in multiple centres (USA, $n=1$; Europe, $n=1$ ). Five articles $^{23172021}(62.5 \%)$ reported adjusted HR. Seven studies $^{2}{ }^{16-21}$ reported patients with ischaemic fibrosis, and the remaining one studies ${ }^{3}$ reported patients with ischaemic and non-ischaemic fibrosis.

Of the 8 eligible studies, 7 received NOS scores between 7 and 9, and the overall mean NOS score was 7.5. Overall, the aforementioned analysis showed that the included articles had high quality (table 1). Among the identified studies, there was no risk of publication bias according to a visual analysis of the funnel plot (online supplemental figure S1).

\section{Prevalence of LGE-MRI-detected myocardial fibrosis and annualised event rates}

Across the 8 studies, the prevalence of myocardial fibrosis detected by LGE-MRI ranged from $15 \%$ to $62 \%$, and the prevalence of LGE-MRI-detected myocardial fibrosis in the total sample was $38.09 \%(n=427)$. Furthermore, a total of 164 events occurred in the diabetes group $(n=1121)$ during the median follow-up of 3.4 years. Patients with diabetes had annualised event rates for MACCEs of $4.3 \%$. Additionally, 3 studies $^{2} 1921$ reported a total of 301 patients with diabetes, and $19.27 \%(\mathrm{n}=58)$ of patients with diabetes had LGE-MRI-detected myocardial fibrosis. Twenty-seven events occurred in these diabetic patients with LGE-MRI-detected myocardial fibrosis over a median follow-up of 3.9 years. The annualised event rate of patients with diabetes and LGE-MRI-detected myocardial fibrosis was $11.94 \%$.

Major adverse cardiac and cerebrovascular events and major adverse cardiac events

A total of 8 studies reported the outcome of MACCEs or MACEs, and the presence of myocardial fibrosis detected 


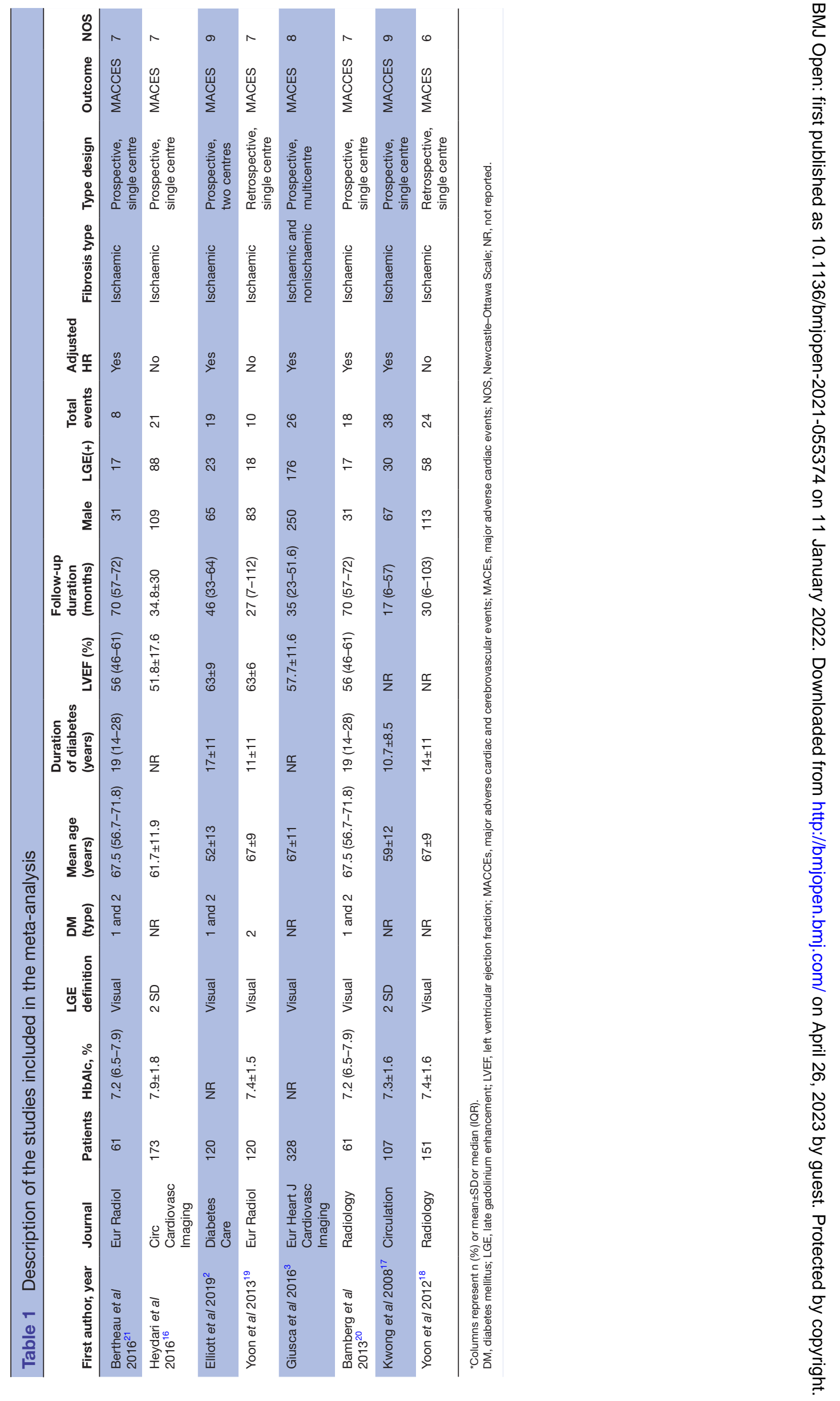


Study

$\%$

ID

$\mathrm{HR}(95 \% \mathrm{Cl})$

Weight

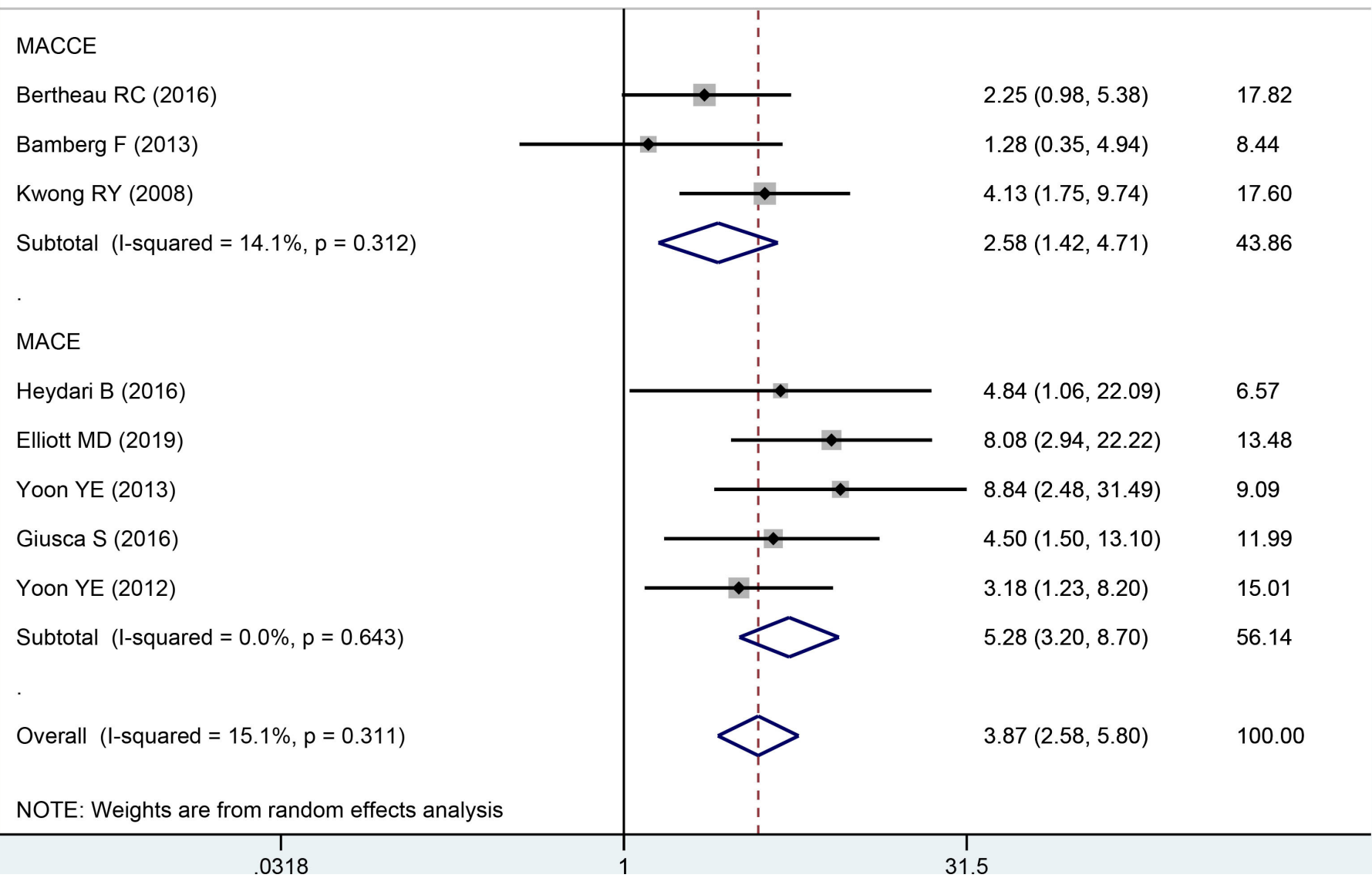

Figure 2 Forest plot and pooled estimates of the effect of myocardial fibrosis detected by LGE on the risk of MACCEs or MACEs. LGE, late gadolinium enhancement; MACCEs, major adverse cardiac and cerebrovascular events; MACEs, major adverse cardiac events.

by LGE-MRI was a strong predictor of MACCEs and MACEs in patients with diabetes (random effects HR 3.87, $95 \%$ CI 2.58 to $5.80 ; \mathrm{p}<0.0001$ ) (figure 2 ). There was low heterogeneity $\left(\mathrm{I}^{2}=15.1 \%, \mathrm{p}=0.311\right)$ in the meta-analysis. In addition, sensitivity analysis performed by excluding one study at a time did not reveal any significant changes in the HR values.

In the analysis of the outcome of MACCEs, 3 articles $^{172021}$ were included in this subgroup analysis, including 64 participants with LGE-MRI-detected myocardial fibrosis and 165 without LGE-MRI-detected myocardial fibrosis, with a total of 64 MACCEs during the follow-up period. Myocardial fibrosis detected by LGEMRI was associated with an increased risk of MACCEs in patients with diabetes. The pooled HR obtained via the random effects model was 2.58 (95\% CI 1.42 to 4.71; $\mathrm{p}=0.002)$, with no evidence of heterogeneity $\left(\mathrm{I}^{2}=14.1 \%\right.$; $\mathrm{p}=0.312$ ) (figure 2).

To explore the association between myocardial fibrosis and the outcome of MACEs in patients with diabetes, we included five articles 23161819 that provided a subgroup outcome analysis of MACEs. The results showed that the presence of LGE-MRI-detected myocardial fibrosis in diabetes was associated with a significantly higher risk of MACEs. As in the discovery analyses, the pooled HR obtained via the random effects model was 5.28 (95\% CI 3.20 to $8.70 ; \mathrm{p}<0.001)$, with no significant heterogeneity $\left(\mathrm{I}^{2}=0 \% ; \mathrm{p}=0.643\right.$ ) (figure 2).

To further verify the robustness of the results, we grouped all included studies by adjusted or non-adjusted HR. In patients with diabetes, myocardial fibrosis detected by LGE-MRI was associated with an increased risk of MACCEs and MACEs in a subgroup analysis with or without adjusted HR. The pooled HRs obtained via a random effects model were 3.52 (95\% CI 2.02 to 6.16 ; $\left.\mathrm{I}^{2}=35.8 \%\right)$ and $4.63\left(95 \%\right.$ CI 2.35 to $\left.9.14 ; \mathrm{I}^{2}=0 \%\right)$, respectively. There was no significant heterogeneity among the studies (online supplemental figure S2).

To evaluate the effects of the myocardial fibrosis pattern, we further calculated a pooled HR by source of diabetes with different patterns of myocardial fibrosis. In patients with diabetes, ischaemic fibrosis detected 


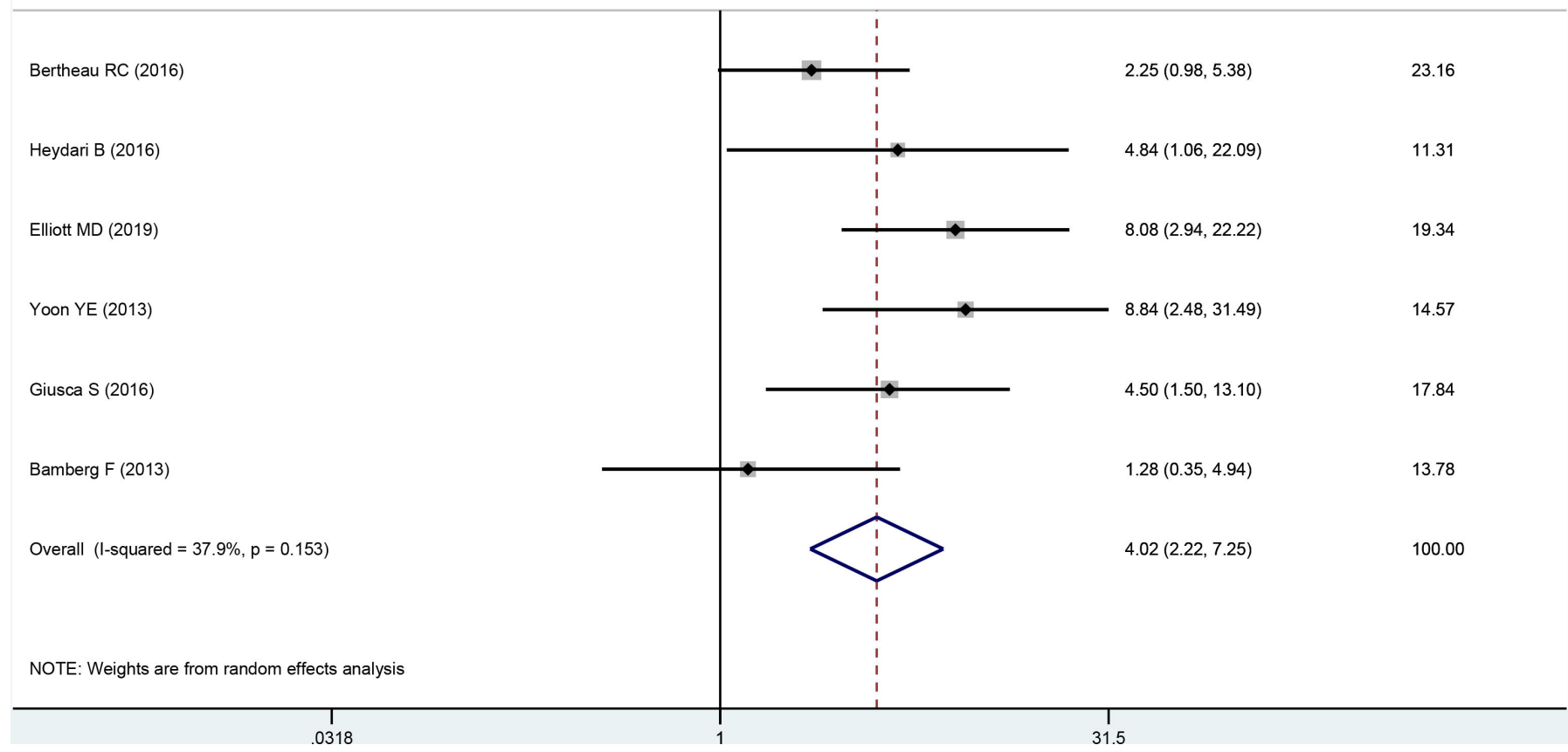

Figure 3 Forest plots of six studies for pooled HR for MACCEs and MACEs in patients with diabetes with normal left ventricular ejection fraction and myocardial fibrosis detected by LGE. LGE, late gadolinium enhancement; MACCEs, major adverse cardiac and cerebrovascular events; MACEs, major adverse cardiac events.

by LGE-MRI was significantly associated with increased MACCEs and MACEs (random effects HR 3.80, 95\% CI 2.38 to $6.07 ; \mathrm{I}^{2}=26.4 \%$ ). No study in our meta-analysis reported the relationship between nonischaemic fibrosis and the risk of MACCEs and MACEs alone; hence, we cannot perform a meta-analysis to assess the relationship between nonischaemic fibrosis and MACCEs/MACEs (online supplemental figure S3).

To confirm whether there were similar results in patients with preserved LV ejection fraction, we conducted a subgroup analysis with six studies. Among individuals with diabetes and LV ejection fraction $>50 \%$, the presence of myocardial fibrosis assessed by LGE-MRI was significantly associated with MACCEs and MACEs. The pooled HR obtained via the random effects model was 3.98 (95\% CI 2.22 to $7.25 ; \mathrm{p}<0.001)$, and there was a medium amount of heterogeneity among the studies $\left(\mathrm{I}^{2}=37.9 \%\right.$; $\left.=0.153\right)$ (figure 3).

\section{DISCUSSION}

In this meta-analysis, the prevalence of myocardial fibrosis (mainly ischaemic fibrosis) assessed by LGE-MRI was increased in patients with diabetes, occurring in $38.09 \%$ of them, and it was associated with an increased risk for MACCEs and MACEs, even when the LV ejection fraction persisted. Moreover, ischaemic myocardial fibrosis detected by LGE-MRI has a higher predictive value for the occurrence of future MACEs than MACCEs in patients with diabetes. However, in this study, the relationship of non-ischaemic LGE-MRI-detected fibrosis and MACCEs/MACEs in patients with diabetes was not elucidated. Therefore, ischaemic myocardial fibrosis by LGEMRI may be an imaging biomarker for predicting adverse outcomes in patients with diabetes.

In our meta-analysis, the results supported previous studies showing that participants with diabetes have a higher presence of myocardial fibrosis detected by LGEMRI, especially ischaemic fibrosis. Importantly, in our included studies, the presence of myocardial fibrosis in symptomatic patients with diabetes was higher than that in asymptomatic patients with diabetes. ${ }^{2317}$ Furthermore, unrecognised ischaemic myocardial fibrosis in patients with diabetes is considered as a biomarker which is responsible for poor outcomes, and maybe provides a stronger prognostic value than conventional cardiovascular risk factors. $^{2}{ }^{17}$ All studies included in our meta-analysis involved patients who had suffered a unrecognised MI, which implied they might represented a higher risk population. Current guidelines recommend that MRI may serve as a risk tool in patients with asymptomatic diabetes 
with moderate or high risk of cardiovascular disease. ${ }^{14}$ However, it is unclear whether LGE-MRI-detected myocardial fibrosis would indicate an increased risk of MACEs in patients with diabetes at low cardiovascular risk. Notably, in our meta-analysis, focal ischaemic myocardial fibrosis detected by LGE-MRI did seem to predict a higher occurrence of MACCEs/MACEs, and the annualised event rate for MACCEs/MACEs in patients with diabetes and LGE-MRI-detected myocardial fibrosis was $11.94 \%$. Additionally, the presence of ischaemic myocardial fibrosis indicated an eightfold higher risk for death/MI even in asymptomatic patients with diabetes. ${ }^{2}$ Notably, other techniques, such as ECG, have lower accuracy and sensitivity for detecting myocardial fibrosis than LGE-MRI. ${ }^{35} 36$ Thus, this finding may highlight the value of LGE-MRI for screening for cardiovascular risk in symptomatic patients with diabetes.

The risk of myocardial fibrosis in patients with diabetes is increased, and there are multiple factors that influence this relationship. First, patients with diabetes have a higher risk for coronary artery disease and myocardial dysfunction. ${ }^{37-39}$ Moreover, hyperglycaemic metabolism, microvascular disease and cardiac autonomic neuropathy are involved in the mechanisms of myocardial fibrosis. ${ }^{40}{ }^{41}$ However, many studies have shown that patients with diabetes have a high incidence of obesity, visceral fat, hyperlipidaemia and insulin resistance, which may impair myocardial function. ${ }^{642}{ }^{43}$ Furthermore, the multiple risk factors described above should increase the myocardial fibrosis burden. In addition, myocardial fibrosis is widespread in subjects with diabetes and may be associated with a high risk for cardiovascular disease.

Although focal myocardial fibrosis translates to an adverse outcome in the future and is not fully clear, several potential mechanisms may lead to MACCEs/MACEs. First, patients with diabetes are more inclined to develop myocardial fibrosis, and myocardial fibrosis is associated with ventricular arrhythmia and heart failure. ${ }^{344-46}$ Second, patients with diabetes and myocardial fibrosis usually have a greater burden of microvascular complications, such as myocardial ischaemia, which confers an increased risk of MACCEs/MACEs. ${ }^{16}{ }^{47}$ Additionally, the myocardial fibrosis detected by LGE-MRI, especially subendocardial fibrosis, indicates that patients with diabetes have had a subendocardial infarction in the past, which denotes a higher risk of MACEs in the future. ${ }^{49}$ Furthermore, subjects with diabetes had higher LV and left atrial remodelling due to myocardial fibrosis. ${ }^{750}$ For these reasons, the myocardial fibrosis detected by LGEMRI has great potential to lead to adverse outcomes in the future.

As previously described, LGE-MRI has become a powerful non-invasive imaging method for the assessment of myocardial fibrosis. ${ }^{11}$ Although two studies ${ }^{2021}$ included in our meta-analysis showed that ischaemic myocardial fibrosis detected by LGE-MRI did not increase the rate of MACCEs, our meta-analysis demonstrated that the presence of ischaemic myocardial fibrosis derived from
LGE-MRI conferred an HR of 3.80 for future MACCEs/ MACEs in individuals with diabetes. This might be explained by the following reasons: limited patient numbers and a higher prevalence of cardiovascular disease at patient enrolment. Indeed, detecting myocardial fibrosis can be used to clinically assess myocardial damage and to stratify cardiovascular risk in participants with diabetes. To date, only one study, which screened for asymptomatic diabetes by LGE-MRI, showed that diabetes with ischaemic myocardial fibrosis conferred an eightfold higher risk for all-cause mortality and MI. ${ }^{2}$ The prevalence of ischaemic myocardial fibrosis detected by LGE-MRI among patients with diabetes is higher than that among patients without diabetes. ${ }^{330}$ Although there were several studies that have reported the prognostic value of ischaemic myocardial fibrosis detected by LGE-MRI in patients with diabetes, the prognostic value of non-ischaemic myocardial fibrosis has not been studied. Therefore, patients with diabetes and ischaemic myocardial fibrosis might need aggressive management of cardiac and cerebrovascular risk factors. Given the scarcity of studies that focused on the prognosis of nonischaemic myocardial fibrosis in patients with diabetes, more relevant studies are needed.

However, our meta-analysis has some limitations. First, in our meta-analysis, two studies ${ }^{2021}$ were from the same group of patients but reported different outcomes. However, when we excluded either of the above articles, the pooled HR and heterogeneity did not change significantly. Second, the incidence of myocardial fibrosis in patients with diabetes was not obtained via communitybased epidemiology research. Therefore, the prevalence of myocardial fibrosis may be higher in this study, which pooled studies including high-risk or averagerisk populations with diabetes. Third, a previous study found that non-ischaemic LGE-MRI-detected myocardial fibrosis is associated with increased myocardial mass, increased myocardial extracellular volume and impaired diastolic parameters. ${ }^{7}$ However, subgroup analysis was not conducted to evaluate the effect of non-ischaemic myocardial fibrosis on MACCEs/MACEs in patients with diabetes due to a lack of information. Further studies are needed to establish non-ischaemic LGE-MRI lesions and their prognosis. Fourth, most studies selected in this meta-analysis reported adjusted HR, and various adjustments for adverse outcomes among the selected studies may affect the pooled results. However, the heterogeneity among the selected studies was low, and publication bias did not exist. This might strengthen the clinical meaning of the pooled result. Finally, the incremental value of diabetes duration to the prevalence and incidence of LGE-MRI-detected myocardial fibrosis was not revealed. However, diabetes duration plays a central role in the assessment of cardiovascular risk. ${ }^{14}{ }^{51}$ Hence, prospective studies that evaluate the association between diabetes duration and myocardial fibrosis and determine the best time to screen myocardial fibrosis by LGE-MRI for risk stratification in patients with diabetes are needed. 


\section{CONCLUSIONS}

In patients with diabetes, the presence of myocardial fibrosis detected by LGE-MRI, especially ischaemic lesions, was markedly associated with an important and increased risk of MACCEs/MACEs. This meta-analysis highlights the potential role of LGE-MRI in helping predict MACCEs/MACEs in complicated patients with diabetes, especially those with cardiac complications and a high risk for myocardial fibrosis. Although we reported that ischaemic myocardial fibrosis detected by LGE-MRI is a strong risk marker for improving risk stratification in patients with diabetes, whether LGE-MRI provides incremental prognostic information with respect to MACCEs/ MACEs over risk stratification by conventional cardiovascular risk factors requires further study.

\section{Author affiliations}

${ }^{1}$ Department of Radiology, Key Laboratory of Birth Defects and Related Diseases of Women and Children of Ministry of Education, Sichuan University West China Second University Hospital, Chengdu, China

${ }^{2}$ Department of Radiology, Chengdu Fifth People's Hospital, Chengdu, Sichuan, China

${ }^{3}$ Department of Radiology, Sichuan University West China Hospital, Chengdu, Sichuan, China

${ }^{4}$ Key Laboratory of Obstetrics \& Gynecology and Pediatric Disease and Birth Defects of Ministry of Education, Sichuan University West China Second University Hospital, Chengdu, China

Contributors $\mathrm{Y}-\mathrm{kG}$ is the guarantor of the integrity of the entire study. ZY and RX conceived of this study, and participated in its design and coordination and drafted the manuscript. Contribution to the conceptualisation and design: J-rW, H-yX, HF, L-jX and M-xY. Data analysis and interpretation: LZ, L-yW, HLi and HLiu. Obtaining funding: Z-gY and Y-kG. Z-gY and Y-kG interpreted the results, critically revised the manuscript, and helped to and approved the final version. All authors read and approved this manuscript.

Funding This work was supported by the National Natural Science Foundation of China (No. 81771887, 81771897, 81971586, 81901712); the Program for Young Scholars and Innovative Research Team in Sichuan Province (No. 2017TD0005) of China; and the 135 project for disciplines of excellence, West China Hospital, Sichuan University (No. ZYGD18013).

Competing interests None declared.

Patient consent for publication Not applicable.

Ethics approval Because this was a review, we did not we did not apply for ethics approval for this article from the institutional ethics review board of West China Second University Hospital, exempted this study. Participants gave informed consent to participate in the study before taking part.

Provenance and peer review Not commissioned; externally peer reviewed.

Data availability statement № data are available.

Supplemental material This content has been supplied by the author(s). It has not been vetted by BMJ Publishing Group Limited (BMJ) and may not have been peer-reviewed. Any opinions or recommendations discussed are solely those of the author(s) and are not endorsed by BMJ. BMJ disclaims all liability and responsibility arising from any reliance placed on the content. Where the content includes any translated material, BMJ does not warrant the accuracy and reliability of the translations (including but not limited to local regulations, clinical guidelines, terminology, drug names and drug dosages), and is not responsible for any error and/or omissions arising from translation and adaptation or otherwise.

Open access This is an open access article distributed in accordance with the Creative Commons Attribution Non Commercial (CC BY-NC 4.0) license, which permits others to distribute, remix, adapt, build upon this work non-commercially, and license their derivative works on different terms, provided the original work is properly cited, appropriate credit is given, any changes made indicated, and the use is non-commercial. See: http://creativecommons.org/licenses/by-nc/4.0/.
ORCID iD

Ying-kun Guo http://orcid.org/0000-0001-8437-9887

\section{REFERENCES}

1 Cho NH, Shaw JE, Karuranga S, et al. IDF diabetes atlas: global estimates of diabetes prevalence for 2017 and projections for 2045. Diabetes Res Clin Pract 2018;138:271-81.

2 Elliott MD, Heitner JF, Kim H, et al. Prevalence and prognosis of unrecognized myocardial infarction in asymptomatic patients with diabetes: a two-center study with up to 5 years of follow-up. Diabetes Care 2019;42:1290-6.

3 Giusca S, Kelle S, Nagel E, et al. Differences in the prognostic relevance of myocardial ischaemia and scar by cardiac magnetic resonance in patients with and without diabetes mellitus. Eur Heart $J$ Cardiovasc Imaging 2016;17:812-20.

4 Jia G, Hill MA, Sowers JR. Diabetic cardiomyopathy: an update of mechanisms contributing to this clinical entity. Circ Res 2018;122:624-38.

5 Russo I, Frangogiannis NG. Diabetes-associated cardiac fibrosis: cellular effectors, molecular mechanisms and therapeutic opportunities. J Mol Cell Cardiol 2016;90:84-93.

6 Marwick TH, Ritchie R, Shaw JE, et al. Implications of underlying mechanisms for the recognition and management of diabetic cardiomyopathy. J Am Coll Cardiol 2018;71:339-51.

7 Storz C, Hetterich H, Lorbeer R, et al. Myocardial tissue characterization by contrast-enhanced cardiac magnetic resonance imaging in subjects with prediabetes, diabetes, and normal controls with preserved ejection fraction from the general population. Eur Heart J Cardiovasc Imaging 2018;19:701-8.

8 Jia G, Whaley-Connell A, Sowers JR. Diabetic cardiomyopathy: a hyperglycaemia- and insulin-resistance-induced heart disease. Diabetologia 2018;61:21-8.

9 Bojer AS, Sørensen MH, Vejlstrup N, et al. Distinct non-ischemic myocardial late gadolinium enhancement lesions in patients with type 2 diabetes. Cardiovasc Diabetol 2020;19:184.

10 Armstrong AC, Ambale-Venkatesh B, Turkbey E, et al. Association of cardiovascular risk factors and myocardial fibrosis with early cardiac dysfunction in type 1 diabetes: the diabetes control and complications Trial/Epidemiology of diabetes interventions and complications study. Diabetes Care 2017;40:405-11.

11 Mewton N, Liu CY, Croisille P, et al. Assessment of myocardial fibrosis with cardiovascular magnetic resonance. J Am Coll Cardiol 2011;57:891-903.

12 Hashimura $\mathrm{H}$, Kimura F, Ishibashi-Ueda $\mathrm{H}$, et al. Radiologicpathologic correlation of primary and secondary cardiomyopathies: MR imaging and histopathologic findings in hearts from autopsy and transplantation. Radiographics 2017;37:719-36.

13 Iles LM, Ellims AH, Llewellyn $\mathrm{H}$, et al. Histological validation of cardiac magnetic resonance analysis of regional and diffuse interstitial myocardial fibrosis. Eur Heart J Cardiovasc Imaging 2015;16:14-22.

14 Cosentino F, Grant PJ, Aboyans V. ESC guidelines on diabetes, prediabetes, and cardiovascular diseases developed in collaboration with the EASD. Eur Heart J 2019;2020:255-323.

15 Jensen MT, Fung K, Aung N, et al. Changes in cardiac morphology and function in individuals with diabetes mellitus: the UK Biobank cardiovascular magnetic resonance substudy. Circ Cardiovasc Imaging 2019;12:e009476.

16 Heydari B, Juan $\mathrm{Y}-\mathrm{H}$, Liu $\mathrm{H}$, et al. Stress perfusion cardiac magnetic resonance imaging effectively risk stratifies diabetic patients with suspected myocardial ischemia. Circ Cardiovasc Imaging 2016;9:e004136.

17 Kwong RY, Sattar $\mathrm{H}$, Wu H, et al. Incidence and prognostic implication of unrecognized myocardial scar characterized by cardiac magnetic resonance in diabetic patients without clinical evidence of myocardial infarction. Circulation 2008;118:1011-20.

18 Yoon YE, Kitagawa K, Kato S, et al. Prognostic significance of unrecognized myocardial infarction detected with MR imaging in patients with impaired fasting glucose compared with those with diabetes. Radiology 2012;262:807-15.

19 Yoon YE, Kitagawa K, Kato S, et al. Prognostic value of unrecognised myocardial infarction detected by late gadolinium-enhanced MRI in diabetic patients with normal global and regional left ventricular systolic function. Eur Radiol 2013;23:2101-8.

20 Bamberg F, Parhofer KG, Lochner E, et al. Diabetes mellitus: longterm prognostic value of whole-body MR imaging for the occurrence of cardiac and cerebrovascular events. Radiology 2013;269:730-7. 
21 Bertheau RC, Bamberg F, Lochner E, et al. Whole-body MR imaging including angiography: predicting recurrent events in diabetics. Eur Radiol 2016;26:1420-30.

22 Stroup DF, Berlin JA, Morton SC, et al. Meta-analysis of observational studies in epidemiology: a proposal for reporting. meta-analysis of observational studies in epidemiology (moose) group. JAMA 2000;283:2008-12.

23 Hutton B, Salanti G, Caldwell DM, et al. The PRISMA extension statement for reporting of systematic reviews incorporating network meta-analyses of health care interventions: checklist and explanations. Ann Intern Med 2015;162:777-84.

24 Stang A. Critical evaluation of the Newcastle-Ottawa scale for the assessment of the quality of nonrandomized studies in metaanalyses. Eur J Epidemiol 2010;25:603-5.

25 Zeng X, Zhang Y, Kwong JSW, et al. The methodological quality assessment tools for preclinical and clinical studies, systematic review and meta-analysis, and clinical practice guideline: a systematic review. J Evid Based Med 2015;8:2-10.

26 Mantovani A, Byrne CD, Bonora E, et al. Nonalcoholic fatty liver disease and risk of incident type 2 diabetes: a meta-analysis. Diabetes Care 2018;41:372-82.

27 Higgins JPT, Thompson SG. Quantifying heterogeneity in a metaanalysis. Stat Med 2002;21:1539-58.

$28 \mathrm{Liu} \mathrm{JL}$. The role of the funnel plot in detecting publication and related biases in meta-analysis. Evid Based Dent 2011;12:121-2.

29 Reinstadler SJ, Stiermaier T, Eitel C, et al. Relationship between diabetes and ischaemic injury among patients with revascularized ST-elevation myocardial infarction. Diabetes Obes Metab 2017;19:1706-13.

30 Lindman BR, Dávila-Román VG, Mann DL, et al. Cardiovascular phenotype in HFpEF patients with or without diabetes: a RELAX trial ancillary study. J Am Coll Cardiol 2014;64:541-9.

31 Eitel I, Hintze S, de Waha S, et al. Prognostic impact of hyperglycemia in nondiabetic and diabetic patients with ST-elevation myocardial infarction: insights from contrast-enhanced magnetic resonance imaging. Circ Cardiovasc Imaging 2012;5:708-18.

32 Donnino R, Patel S, Nguyen $\mathrm{AH}$, et al. Comparison of quantity of left ventricular scarring and remodeling by magnetic resonance imaging in patients with versus without diabetes mellitus and with coronary artery disease. Am J Cardiol 2011;107:1575-8.

33 Lejeune S, Roy C, Slimani A, et al. Diabetic phenotype and prognosis of patients with heart failure and preserved ejection fraction in a real life cohort. Cardiovasc Diabetol 2021;20:48.

34 Kato S, Fukui K, Kodama S, et al. Incremental prognostic value of coronary flow reserve determined by phase-contrast cine cardiovascular magnetic resonance of the coronary sinus in patients with diabetes mellitus. J Cardiovasc Magn Reson 2020;22:73.

35 Ramos R, Albert X, Sala J, et al. Prevalence and incidence of Q-wave unrecognized myocardial infarction in general population: diagnostic value of the electrocardiogram. The REGICOR study. Int J Cardiol 2016;225:300-5

36 Barbier CE, Bjerner T, Johansson L, et al. Myocardial scars more frequent than expected: magnetic resonance imaging detects potential risk group. J Am Coll Cardiol 2006;48:765-71.
37 Bertoni AG, Goff DC, D'Agostino RB, et al. Diabetic cardiomyopathy and subclinical cardiovascular disease: the multi-ethnic study of atherosclerosis (MESA). Diabetes Care 2006;29:588-94.

38 Shivu GN, Phan TT, Abozguia K, et al. Relationship between coronary microvascular dysfunction and cardiac energetics impairment in type 1 diabetes mellitus. Circulation 2010;121:1209-15.

39 Campbell DJ, Somaratne JB, Jenkins AJ, et al. Impact of type 2 diabetes and the metabolic syndrome on myocardial structure and microvasculature of men with coronary artery disease. Cardiovasc Diabetol 2011;10:80.

40 Tarquini R, Lazzeri C, Pala L, et al. The diabetic cardiomyopathy. Acta Diabetol 2011;48:173-81.

41 Gao Y, Yang Z-G, Ren Y, et al. Evaluation of myocardial fibrosis in diabetes with cardiac magnetic resonance T1-mapping: correlation with the high-level hemoglobin A1c. Diabetes Res Clin Pract 2019;150:72-80.

42 Turkbey EB, Backlund J-YC, Genuth S, et al. Myocardial structure, function, and scar in patients with type 1 diabetes mellitus. Circulation 2011;124:1737-46.

$43 \mathrm{Ng} \mathrm{ACT}$, Strudwick M, van der Geest RJ, et al. Impact of epicardial adipose tissue, left ventricular myocardial fat content, and interstitial fibrosis on myocardial contractile function. Circ Cardiovasc Imaging 2018;11:e007372.

44 Anselmino M, Matta M, D'ascenzo F, et al. Catheter ablation of atrial fibrillation in patients with diabetes mellitus: a systematic review and meta-analysis. Europace 2015;17:1518-25.

45 Gulsin GS, Kanagala P, Chan DCS, et al. Differential left ventricular and left atrial remodelling in heart failure with preserved ejection fraction patients with and without diabetes. Ther Adv Endocrinol Metab 2019;10:2042018819861593.

46 Mordi I, Bezerra H, Carrick D, et al. The combined incremental prognostic value of LVEF, late gadolinium enhancement, and global circumferential strain assessed by CMR. JACC Cardiovasc Imaging 2015;8:540-9.

47 Sandesara PB, O'Neal WT, Kelli HM, et al. The prognostic significance of diabetes and microvascular complications in patients with heart failure with preserved ejection fraction. Diabetes Care 2018;41:150-5

48 Schelbert EB, Cao JJ, Sigurdsson S, et al. Prevalence and prognosis of unrecognized myocardial infarction determined by cardiac magnetic resonance in older adults. JAMA 2012;308:890-6.

49 Acharya T, Aspelund T, Jonasson TF, et al. Association of unrecognized myocardial infarction with long-term outcomes in community-dwelling older adults: the Iceland MI study. JAMA Cardiol 2018;3:1101-6.

50 Cao Y, Zeng W, Cui Y, et al. Increased myocardial extracellular volume assessed by cardiovascular magnetic resonance $\mathrm{T} 1$ mapping and its determinants in type 2 diabetes mellitus patients with normal myocardial systolic strain. Cardiovasc Diabetol 2018;17:7.

51 Dabelea D, Stafford JM, Mayer-Davis EJ, et al. Association of type 1 diabetes vs type 2 diabetes diagnosed during childhood and adolescence with complications during teenage years and young adulthood. JAMA 2017;317:825-35. 